Sādhanā, Vol. 26, Part 4, August 2001, pp. 371-377. (C) Printed in India

\title{
Effect of dynamic visco-elasticity on vertical and torsional vibrations of a half-space
}

\author{
PRAKASH CHANDRA PAL \\ Department of Applied Mathematics. Indian School of Mines, Dhanbad \\ 826004 , India \\ e-mail: pcpal_ism@yahoo.co.in
}

MS received 7 September 1999; revised 21 March 2001

\begin{abstract}
By expressing the dynamic visco-elastic characteristic of a material in terms of the complex shear modulus, the vertical vibrations of a visco-elastic half-space as well as that of a mass in visco-elastic half-space are considered here. Torsional vibrations of a visco-elastic half-space is also considered. Numerical results are derived for two cases and shown graphically.
\end{abstract}

Keywords. Visco-elasticity; complex moduli; torsional vibrations; tangential displacement.

\section{Introduction}

A literature survey reveals that the concept of complex moduli allows the solution of problems of dynamic visco-elasticity for stationary vibrations. It can be shown that if the initial conditions are zero and Fourier integral transformation is used, such formulation of the visco-elastic behaviour may be applied to the problems of non-periodic vibrations also.

The determination of stresses and displacements in an isotropic, homogeneous elastic medium due to torsional oscillation has been a subject of considerable interest in solid mechanics and applied mathematics. In particular, torsional vibration of a anisotropic visco-elastic material is largely used in measuring shear constants, shear velocities and shear elasticity of liquids.

Vertical vibrations of an isotropic elastic half-space subjected to a harmonically variable normal load acting over a circular area were treated by Reissner (1936) and later, Bycroft (1956). Reissner \& Sagoci (1944) investigated torsional oscillations in an elastic half-space under the action of periodic shear stress applied to a circular portion of the surface of the half-space. Erguven (1988) considered the dynamical Reissner-Sagoci problem for a radially non-homogeneous material. Pal \& Kumar (1995) showed the effect of inhomogeneity on torsional impulse motion over a circular region in a transversely isotropic elastic half space. Recently, Pal (2000) considered the problem of torsional body forces in visco-elastic half-space.

In this paper I present the results of analysis of the dynamic response of a visco-elastic half-space applying the concept of complex moduli as considered by Snowdon (1978) 
and later by Golden (1982). I obtain the vertical and torsional disturbances and show their nature graphically.

\section{Formulation and solution of the problem}

The dynamic visco-elastic characteristics of a material may be expressed in terms of the complex shear modulus $G_{\omega, T}^{*}$ and the volume modulus $B_{\omega, T}^{*}$ according to the formulae:

$$
\begin{gathered}
G_{\omega, T}^{*}=G_{\omega, T}\left(1+i \delta G_{\omega, T} \operatorname{Sgn} \omega\right), \\
B_{\omega, T}^{*}=B_{\omega, T}\left(1+i \delta B_{\omega, T} \operatorname{Sgn} \omega\right) .
\end{gathered}
$$

Suffixes $\omega, T$ relate the values of complex moduli to a given frequency $\omega$ and temperature $T$. In the present problem, they will be omitted. $\delta$ is the damping parameter.

The concept of complex moduli allows solution of the problems of dynamic visco-elasticity for stationary vibrations. Here we shall consider three different cases.

\subsection{Case I - Vertical vibrations of a visco-elastic half-space}

For an axi-symmetric problem given in cylindrical polar coordinates, the equation of motion will be in the form:

$$
\begin{gathered}
\rho \frac{\partial^{2} u}{\partial t^{2}}=\left(B^{*}+\frac{u}{3} G^{*}\right)\left(\frac{\partial^{2} u}{\partial r^{2}}+\frac{1}{r} \frac{\partial u}{\partial r}-\frac{u}{r^{2}}+\frac{\partial^{2} w}{\partial r \partial z}\right) \\
+G^{*}\left(\frac{\partial^{2} u}{\partial z^{2}}-\frac{\partial^{2} w}{\partial r \partial z}\right), \\
\rho \frac{\partial^{2} w}{\partial t^{2}}=\left(B^{*}+\frac{4}{3} G^{*}\right)\left(\frac{\partial^{2} u}{\partial r \partial z}+\frac{1}{r} \frac{\partial u}{\partial z}-+\frac{\partial^{2} w}{\partial z}\right) \\
+G^{*}\left(\frac{\partial^{2} w}{\partial r^{2}}+\frac{1}{r} \frac{\partial w}{\partial r}-\frac{\partial^{2} u}{\partial r \partial z}\right),
\end{gathered}
$$

where $u(r, z, t)$ is a horizontal displacement, $w(r, z, t)$ is a vertical displacement and $\rho$ the density. We assume the vibration to be harmonic and stationary and so we can write

$$
\begin{aligned}
u(r, z, t) & =u(r, z) e^{i \omega t}, \\
w(r, z, t) & =w(r, z) e^{i \omega t} .
\end{aligned}
$$

We define the Hankel integral transformation as

$$
\begin{aligned}
& u_{H}(\alpha, z)=\int_{0}^{\alpha} r u(r, z) J_{1}(\alpha r) \mathrm{d} r, \\
& w_{H}(\alpha, z)=\int_{0}^{\alpha} r w(r, z) J_{0}(\alpha r) \mathrm{d} r,
\end{aligned}
$$

$u_{H}, w_{H}$ are displacement transforms. We assume

$$
u_{H}=\alpha\left(B^{*}+\frac{4}{3} G^{*}\right) \frac{\mathrm{d}}{\mathrm{d} z} F,
$$




$$
u_{H}=\left[\rho \omega^{2}-\left(B^{*}+\frac{4}{3} G^{*}\right) \alpha^{2}+G^{*} \frac{\mathrm{d}}{\mathrm{d} z}\right] F,
$$

where $F$ is an auxiliary function and assumptions lead to differential equation:

$$
\begin{aligned}
\frac{\mathrm{d}^{4} F}{\mathrm{~d} z^{4}} & +\frac{\mathrm{d}^{2} F}{\mathrm{~d} z^{2}}\left[2 \alpha^{2}+\omega^{2}\left(\frac{1}{c_{1}^{* 2}}+\frac{1}{c_{2}^{* 2}}\right)\right] \\
& +F\left[\alpha^{4}-\omega^{2} \alpha^{2}\left(\frac{1}{c_{1}^{* 2}}+\frac{1}{c_{2}^{* 2}}\right)+\left(\frac{\omega^{4}}{c_{1}^{* 2} c_{2}^{* 2}}\right)\right]=0,
\end{aligned}
$$

where $c_{1}^{*}=\left[\left(B^{*}+(4 / 3) G^{*}\right) / \rho\right]=$ complex velocity of the dilatational wave and $c_{2}^{*}=$ $\left(G^{*} / \rho\right)=$ complex velocity of transverse wave.

Then $F$ can be written as

$$
\begin{aligned}
F(z) & =A_{1} e^{q *}+A_{2} e^{-q * z}+A_{3} e^{s * z}+A_{4} e^{-s * z}, \\
q^{*} & =\left(\alpha^{2}-\left(\omega^{2} / c_{1}^{* 2}\right)\right)^{1 / 2}, s^{*}=\left(\alpha^{2}-\left(\omega^{2} / c_{1}^{* 2}\right)\right)^{1 / 2},
\end{aligned}
$$

and $A_{1}, A_{2}, A_{3}, A_{4}$. are arbitrary complex functions of parameter $\alpha$.

2.1a Boundary conditions: We shall assume a normal load applied to a circular area of radius $a$ distributed uniformly. The boundary conditions on $z=0$ will be

$$
\begin{aligned}
\sigma_{z} & = \begin{cases}P_{0} e^{i \omega t}, & 0<r<a, \\
0, & r>a,\end{cases} \\
\tau_{r z} & =0
\end{aligned}
$$

After obtaining the Hankel integral transform of (8) and simplifying, we have the transform of the vertical displacement $w_{H}$ given by the equations,

$$
\begin{aligned}
w_{H} & =\left\{\left[\left(s^{* 2}+\alpha^{2}\right) e^{q * z}-2 \alpha^{2} e^{-s * z}\right] / \alpha D^{*}\right\} q^{* 2} P_{0} a J_{1}(\alpha \omega), \\
D^{*} & =G^{*} q^{*}\left[\left(2 \alpha^{2}-\left(\omega^{2} / C_{2}^{* 2}\right)\right)^{2}-4 \alpha^{2} q^{*} s^{*}\right],
\end{aligned}
$$

For practical use of the deduced formula some simplification may be adopted. It will be assumed that

$$
\delta_{B}=\delta_{G}=\delta,
$$

and that Poissons ratio $v$ is independent of the frequency. The original vertical deflection $w$ is now given in the form

$$
\begin{aligned}
\frac{w G}{P_{0} a} & =\int_{0}^{\alpha}\left\{-\Omega^{2}\left(\eta^{2}-\frac{\Omega^{2} \zeta}{1+i \delta}\right)^{1 / 2} J_{1}(\eta) J_{0}\left(n \frac{r}{a}\right) \mathrm{d} n\right\} \div \\
& \left\{(1+i \delta)^{2}\left[\left(2 \eta^{2}-\frac{\Omega^{2}}{1+i \delta}\right)^{2}-4 \eta^{2}\left(\eta^{2}-\frac{\Omega^{2} \zeta}{1+i \delta}\right)^{1 / 2}\left(\eta^{2}-\frac{\Omega^{2}}{1+i \delta}\right)^{1 / 2}\right]\right\},
\end{aligned}
$$

when the dimensionless frequency is defined by

$$
\Omega=\omega a / c_{2}, \eta=\alpha a,
$$




$$
\zeta=(1-2 v) /[2(1-v)],
$$

$c_{2}=(G / \rho)^{1 / 2}$ is the velocity of shear waves, where $G$ is the real part of the complex shear modulus.

2.1b Deflection on the surface $z=0$ : We give an example to compute deflection $w(r, 0, t)$ according to (10) i.e. for the deflection on the surface due to uniform distribution of normal load $P$ over a circular area in the form,

$$
w(r, 0, t)=(P / a G)\left(w_{R}+i w_{j}\right) e^{i \omega t} .
$$

Here $w_{R}$ is the real part, $w_{j}$ is the imaginary part, $w_{A}=\left(w_{R}^{2}+w_{j}^{2}\right)^{1 / 2}$ is the amplitude and phase angle $\phi=\tan ^{-1}\left(w_{j} / w_{R}\right)$.

\subsection{Case II - Vertical vibrations of a mass in visco-elastic half-space}

In this case the equation of motion for vertical vibrations of mass $m$ with a circular contact area of radius $a$ is given by

$$
m\left(\partial^{2} w / \partial t^{2}\right)+R(t)=P_{0} e^{i \omega t} .
$$

$R(t)$ is the response of visco-elastic half-space. Here $\bar{w}$ is used as average vertical dynamic deflection. Following Erguven (1988) and using (10), $\bar{w}$ is obtained in the form

$$
\bar{w}=\frac{-2 R}{a G T} \int_{0}^{\alpha} f(\eta, \Omega, \delta, \zeta) J_{1}^{2}(\eta) \mathrm{d} \eta,
$$

where

$$
\begin{aligned}
& f(n, \Omega, \delta, \zeta)=\left\{-\Omega^{2}\left(1-\frac{\Omega^{2} \zeta}{1+i \delta}\right)^{1 / 2}\right\} \div \\
& \quad\left\{(1-i \delta)^{2} \eta\left[\left(2 \eta^{2}-\frac{\Omega^{2}}{1+i \delta}\right)^{2}-4 \eta^{2}\left(\eta^{2}-\frac{\Omega^{2} \zeta}{1+i \delta}\right)^{1 / 2}\left(\eta^{2}-\frac{\Omega^{2}}{1+i \delta}\right)^{1 / 2}\right]\right\} .
\end{aligned}
$$

In general, the average dynamic deflection due to a loading by reaction of the subsoil $R(t)=R e^{i} \omega t$ may be written in the form

$$
\bar{w}=-(R / a b)\left(\bar{w}_{R}+i \bar{w} j\right) e^{i \omega t} .
$$

From (12), $R$ is given by

$$
R=P_{0} /\left\{1+b \Omega^{2} \bar{w}_{R}+i b \Omega^{2} \bar{w}_{j}\right\},
$$

$b$ is the dimensionless parameter given by $b=m / \rho a 3$.

The amplitude-frequency characteristic is given by

$$
|\bar{w}|=\frac{P_{0}}{a G}\left[\frac{\bar{w}_{R}^{2}+\bar{w}_{j}^{2}}{\left(!+b \Omega^{2} \bar{w}_{R}\right)^{2}+\left(b \Omega^{2} w_{j}\right)^{2}}\right]^{1 / 2} .
$$




\subsection{Case III - Torsional vibrations of visco-elastic half-space}

A similar procedure as for vertical vibration may be used for the analysis of torsional vibrations of visco-elastic half-space. The equation of motion has the form

$$
\frac{\partial^{2} u_{\theta}}{\partial r^{2}}+\frac{1}{r} \frac{\partial u_{\theta}}{\partial r}-\frac{u_{\theta}}{r^{2}}+\frac{\partial^{2} u_{\theta}}{\partial z^{2}}=\frac{\rho}{G^{*}} \frac{\partial^{2} u_{\theta}}{\partial t^{2}},
$$

$G^{*}$ is the complex shear modulus of elasticity, $u_{\theta}$ is the tangential displacement.

The shear stresses are given by

$$
\tau_{z \theta}=G^{*} \frac{\partial u_{\theta}}{\partial z}, \tau_{r \theta}=G^{*}\left(\frac{\partial u_{\theta}}{\partial r}-\frac{u_{\theta}}{r}\right) .
$$

We assume stationary harmonic vibrations and hence

$$
u_{\theta}=u_{\theta}(r, z) e^{i \omega t} \text {. }
$$

Now using the Hankel integral transformation, the solution of (17) is

$$
u_{\theta, H}=U_{1}\left(\zeta^{*}\right) e^{\zeta * z}+U_{2}(\zeta *) e^{\zeta * z}
$$

where $\zeta^{*}=\left(\alpha^{2}-\left(\omega^{2} / C_{2}^{* 2}\right)\right)^{1 / 2}$.

2.3a Boundary conditions: The boundary conditions on the surface $z=0$ are

$$
\begin{aligned}
& \tau_{z \theta}=\left(\tau_{0} / a\right) r, 0<r<a, \\
& \tau_{z \theta}=0, \quad r>a .
\end{aligned}
$$

Using boundary condition (19) and in view of physical meaning of the problem only the term with a negative exponent is used, we have for $z=0, u_{\theta, H}$ is given by

$$
u_{\theta, H}=-\left(\tau_{0} a / G^{*}\right)\left(J_{2}(\alpha a) / \alpha \zeta^{*}\right) e^{-\xi z^{*}} .
$$

The displacement on the surface of the half-space for $z=0$ after rearrangements and use of dimensionless quantities $\eta=\alpha a, \Omega=\omega a / c_{2}$ is given by

$$
u_{\theta}=-\frac{\tau_{0} a}{G} \int_{0}^{\alpha} \frac{J_{2}(\eta) J_{1}(\eta(r / a) \mathrm{d} n)}{\left[\eta^{2}(1+i \delta)^{2}-\Omega^{2}(1+i \delta)\right]^{1 / 2}}
$$

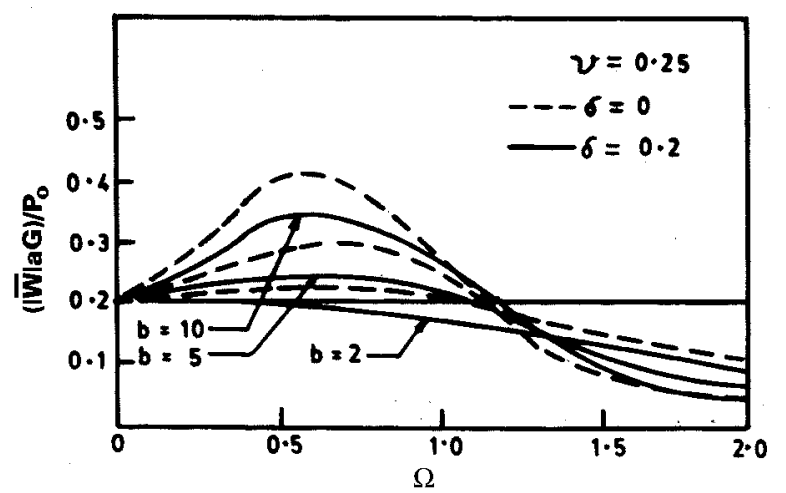

Figure 1. Amplitude frequency characteristic for vertical vibration. 


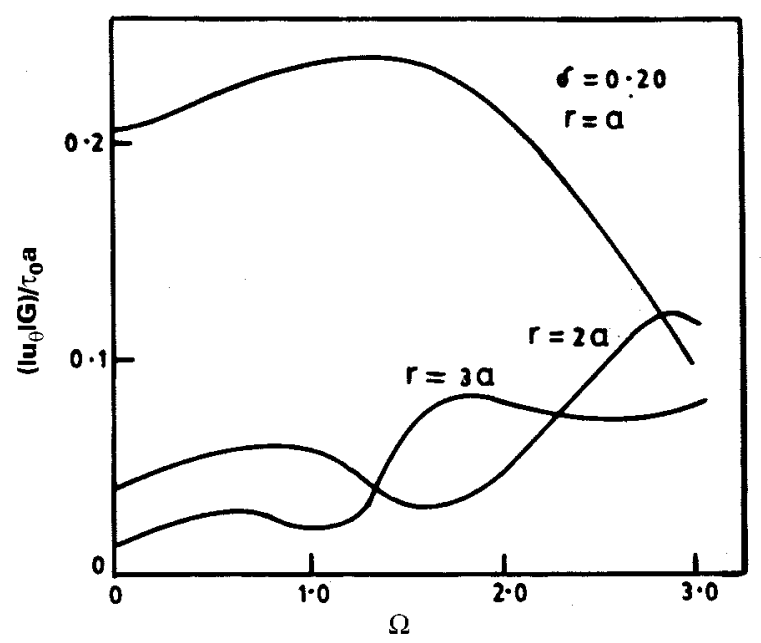

Figure 2. Tangential displacement by torsional vibrations (characteristics).

\section{Numerical results and discussions}

Numerical results are calculated for case II and case III only. Figure 1 shows the computed amplitude-frequency characteristics for vertical vibration of a mass on visco-elastic halfspace. The variations are shown for $v=0.25, \delta=0.2$ for different values of parameter $b-2,5,10$. The dashed curves are for the elastic half-space case. Figure 2 shows the variation of dimensionless absolute value of the tangential displacement $\left(\left|u_{\theta}\right| G\right) / \tau_{0} a$ with the frequency $\Omega$ for $r=a, 2 a, 3 a$. From figure 1 it is inferred that the damping due to internal friction of the subsoil material is not negligible. Figure 2 shows that as we increase the radius of circular contact area, torsional vibrations increases with frequency $\Omega$.

\section{Conclusion}

The ongoing procedures and results of the analysis of a dynamic response of a visco-elastic half-space show that from the point of view of practical applications of dynamic viscoelasticity the most acceptable form of the definition of visco-elastic behaviour of materials seems to be the concept of complex moduli. It is in agreement with the contemporary trend of development and use of numerical methods and computer techniques and at the same time is the real basis for the possibility of the determination of dynamic visco-elastic characteristics of materials in a wide range of frequencies.

\section{References}

Bycroft G N 1956 Forced vibration of a rigid circular plate on a semi-infinite elastic space and on an elastic stratum. Philos. Trans. R. Soc. A248: 327-368

Erguven M E 1988 An axisymmetric fundamental solution and the Reissner Sagoci problem for an internally loaded non-homogeneous transversely isotropic half-space. Int. J. Eng. Sci. 26: $77-84$

Golden J M 1982 Approximate analytic treatment of the problem of a moving ellipsoidal punch on a visco-elastic half-space. Q. J. Mech. Appl. Math. 35: 155-171 
Pal P C, Kumar L 1995 Generation and propagation of SH-type waves due to stress discontinuity in a linear visco-elastic layered medium. Proc. Indian Acad. Sci. (Math. Sci.). 105: 241-249

Pal P C 2000 A note on the torsional body forces in a visco-elastic half-space. Indian J. Pure Appl. Math. 31: 207-213

Reissner E 1936 Axisymmetric stationary vibration of a circular plate on an isotropic elastic half-space subjected to a harmonically variable mass load. Ing. Arch. 7: 838-849

Reissner E, Sagoci H F 1944 Forced torsional oscillations of an elastic half-space. J. Appl. Phys. 15: 652-654

Snowdon J C 1978 Vibrations and shock in damped mechanical system (New York: J. Wiley-Sons) p. 486 